\title{
Prenatal iron deficiency and monoamine oxidase A (MAOA) polymorphisms: combined risk for later cognitive performance in rhesus monkeys
}

\author{
Mari Golub · Casey Hogrefe
}

Received: 4 October 2013/Accepted: 20 December 2013/Published online: 9 January 2014

(C) Springer-Verlag Berlin Heidelberg 2014

\begin{abstract}
Monoamine oxidase A (MAOA) gene polymorphisms resulting in high and low transcription rates are associated with individual differences in reward efficacy and response inhibition. Iron deficiency (ID) is the most frequent single-nutrient deficiency worldwide, and prenatal ID has recently been shown to carry a risk for lower mental development scores in infants. In this study, a potential interaction of MAOA genotype and prenatal ID was studied in young male rhesus monkeys. Cognitive tasks, including problem solving, responsiveness to reward and attention, were used to characterize the potential interaction of these two fetal risks. ID was induced by feeding rhesus monkey dams an iron-deficient (10 ppm, ID) or an iron-sufficient (100 ppm, IS) diet during gestation $(n=10$ / group). Subgroups of the ID and IS diet offspring had lowMAOA or high-MAOA transcription rate polymorphisms. ID combined with low-MAOA genotype showed distinctive effects on reward preference and problem solving while ID in hi-MAOA juveniles modified response inhibition. Given the incidence of ID and MAOA polymorphisms in humans, this interaction could be a significant determinant of cognitive performance.
\end{abstract}

Electronic supplementary material The online version of this article (doi:10.1007/s12263-013-0381-3) contains supplementary material, which is available to authorized users.

M. Golub

Department of Environmental Toxicology, University

of California, Davis, Davis, CA 95616, USA

M. Golub $(\bowtie) \cdot$ C. Hogrefe

California National Primate Research Center (CNPRC),

BMB, University of California,

Davis, One Shields Ave, Davis, CA 95616, USA

e-mail: msgolub@ucdavis.edu
Keywords Rhesus - Infant · Anemia - MAOA polymorphism $\cdot$ Learning $\cdot$ Reward $\cdot$ Attention

\section{Introduction}

With the increased availability of genotyping, the interaction between genetic variation and environment has become more amenable to research. In one area of research, monoamine oxidase A (MAOA) gene polymorphisms in humans appear to interact with adverse childhood environments to permanently alter behavior in ways that are important to social and affective competence (KimCohen et al. 2006; Kinnally et al. 2009; Derringer et al. 2010; Cicchetti et al. 2012; Fergusson et al. 2012). More recently, MAOA interactions with adverse pregnancy life events have been associated with newborn negative emotionality (Hill et al. 2013). Altered monoamine systems in developing brain are implicated as mechanisms in the effects of MAOA genotype on behavior because metabolic degradation of the monoamine neurotransmitters serotonin, dopamine and norepinephrine is influenced by MAOA. To our knowledge, interactions between MAOA polymorphisms and developmental nutrition have not been studied.

We have established rhesus monkey models for prenatal iron deficiency (ID) in order to provide experimental designs that inform human research in this area (Golub et al. 2005a, b, 2006, 2007, 2009, 2012). Prenatal iron deficiency has recently been associated with lower mental development scores in human infants (Chang et al. 2013). Non-human primates are particularly appropriate animal models for the influence of nutritional deficiency on human third trimester brain development. Complex maternal-fetal regulation of nutrient supply is a hallmark of late pregnancy in primates that is not represented in precocial laboratory rodents. 
Using a variety of social challenge tests of young monkeys (Golub et al. 2012), we found that MAOA genotype interacts with ID occurring in utero during fetal development. These young monkeys were not iron deficient at any time during their postnatal development. Our previous studies with prenatal ID in monkeys found little indication of effects on learning and memory, but task performance differences suggested effects on reward and response inhibition (Golub et al. 2007). Because of associations of MAOA polymorphism with reward and response inhibition (Nymberg et al. 2013), we hypothesized that MAOA genotype interaction with developmental ID might emerge in performance of cognitive tasks that rely on these brain systems.

Our test battery was based on methods used in humans and non-human primates to assess the integrity of brain functions important in daily life during childhood, including problem solving, impulsivity and reward sensitivity. These tests were conducted in the same cohort of monkeys previously examined for social responsiveness (Golub et al. 2012).

\section{Method}

Compliance with ethics guidelines

Animal husbandry followed the Guide for the Care and Use of Laboratory Animals of the National Research Council. All protocols were approved prior to implementation by the UC Davis Institutional Animal Care and Use Committee. The California National Primate Research Center (CNPRC) is accredited by the Association for Assessment and Accreditation of Laboratory Animal Care. Animal husbandry and veterinary medicine procedures were performed by specialized staff with advanced training in these areas.

Subjects, diets and genotyping

Pregnant rhesus (Macaca mulatta) dams were assigned to the study after screening for reproductive history. Experimental groups were balanced for dam age, weight and parity. Monkey dams were time mated and fed an iron-deficient $(10 \mathrm{ppm}, \mathrm{ID}, n=10)$ or an iron-sufficient (100 ppm, IS, $n=10$ ) diet during gestation (165 days) beginning at pregnancy identification at gestation day $35-45$ by ultrasound. Fetal sex was identified from maternal blood samples (Jimenez and Tarantal 2003), and only pregnancies with male fetuses were entered into the experiment. Diet composition, feeding schedules and animal husbandry have been reported (Golub et al. 2012). After birth, experimental diets were discontinued and male infants were reared by their
Table 1 Infant background information

\begin{tabular}{lcr}
\hline & IS $(n=10)$ & ID $(n=10)$ \\
\hline Weight $(\mathrm{kg})$ & & \\
Birth & $0.60 \pm 0.03^{\mathrm{a}}$ & $0.55 \pm 0.03$ \\
12 months & $2.37 \pm 0.06$ & $2.21 \pm 0.04$ \\
Crown-rump $(\mathrm{mm})$ & & \\
Birth & $200 \pm 3$ & $196 \pm 2$ \\
12 months & $355 \pm 5$ & $347 \pm 2$ \\
Head circumference $(\mathrm{mm})$ & \\
Birth & $204.9 \pm 1.8$ & $200.2 \pm 2.6$ \\
12 months & $25.3 \pm 0.2$ & $24.8 \pm 0.2$ \\
Hemoglobin $(\mathrm{g} / \mathrm{dL})$ & & \\
Birth & $18.5 \pm 0.9$ & $19.1 \pm 0.8$ \\
12 months & $12.5 \pm 0.2$ & \\
Ferritin (ng/mL) & & $28.6 \pm 6.3$ \\
Birth & & $16.3 \pm 2.1$ \\
12 months & $58.2 \pm 14.1$ & \\
\hline
\end{tabular}

There were no statistically significant group differences

${ }^{\mathrm{a}}$ Mean \pm SEM

${ }^{\mathrm{b}} n=9 /$ group

mothers in double cages with another mother-infant pair until weaning at 5-6 months of age. They were then caged with a same-sex, like-age peer during a 2-year assessment battery (Golub et al. 2012) including tests reported here (see Online Resource 1). During the test period, groups did not differ in growth or iron status (Table 1).

Genotypes based on VNTR polymorphisms in the upstream regulatory region of the rhesus MAOA gene (rhMAOA-LPR) were obtained from routine genotyping conducted at CNPRC (Newman et al. 2005; Capitanio et al. 2012; Golub et al. 2012). Infants in our study were identified as hemizygous for low-MAOA (7 VNTR) or hiMAOA (4, 5 or 6 VNTR) polymorphisms resulting in four groups: hi-MAOA IS $(n=5)$, hi-MAOA ID $(n=5)$, lowMAOA IS $(n=5)$ and low-MAOA ID $(n=4)$. One infant (in the ID group) was excluded from the study due to an ambiguous MAOA VNTR.

\section{Problem solving (16-18 months of age)}

Previous testing of juveniles with trial-based learning in the Wisconsin General Testing Apparatus (WGTA) did not show effects of prenatal ID on learning and memory (Golub et al. 2007). The present study used a less structured test platform, problem solving in the home cage. Monkeys received one problem each weekday between 16 and 18 months of age. Finger mazes and puzzle boxes were presented on alternate days for 45-min sessions. For the puzzle box test, fresh food rewards (carrots, apple slices, grapes) were placed inside clear plexiglass boxes 
$(20.3 \times 10.2 \times 7.6 \mathrm{~cm})$ with hinged lids that were closed and secured with nine different hardware closures (latches and clasps) of increasing complexity (shown in Online Resource 2). The monkey needed to undo the closures in order to open the box and retrieve the rewards, a task requiring trial-and-error manipulation of the closures. Commercial finger mazes (Primate Products, Inc., Immokalee, FL) had removable inserts that could be placed to create 11 increasingly complex configurations (Tsuchida et al. 2003). The monkey used a finger to move three pieces of carrot or apple through the maze to a trough where they could be retrieved, a task that required persistence and concentration. Criterion for each problem was retrieval of the rewards on two successive days.

\section{Reward assessment tests}

Earlier work using the prenatal ID monkey model suggested effects of ID on a delayed reward task (Golub et al. 2007). Reward sensitivity is also a brain system implicated in the social and conduct disorders associated with human low-MAOA polymorphisms (Buckholtz et al. 2008). Three tests directed at sensitivity of reward systems were included in the test battery.

\section{Reward devaluation test (8-11 months of age)}

The reward devaluation test (Malkova et al. 1997; Machado and Bachevalier 2007; West et al. 2011) was conducted in the WGTA and consisted of three parts: concurrent discrimination learning, preferred reward devaluation series and nonpreferred reward devaluation series. For concurrent discrimination, 12 pairs of objects were presented during a 12-trial session, with one object of each pair designated as correct. A food reward could be obtained by displacing the correct object to uncover a food well. Half the correct choices were rewarded with raisins and half with Fruity Gems ${ }^{\circledR}$ (Bioserv, Frenchtown, NJ). (The Fruity Gem reward was replaced with marshmallow for two monkeys.) Discrimination training continued to either a criterion of $85 \%$ correct in five successive sessions, or 60 sessions, whichever came first. After this, raisin devaluation and Fruity Gem devaluation series were conducted. Each series consisted of four sessions: a discrimination session identical to previous testing, a non-satiation preference session which consisted of trials pairing a correct object rewarded with raisins with a correct object rewarded with Fruity Gems, a satiation session in which the animals were given free access to one of the two rewards in their home cage for 25 min prior to a preference session and a final post-satiation preference session. During preference sessions, the number of choices of objects associated with each food reward was recorded. All trials were 30 s long with a 30-s inter-trial interval.
Reward delay test (11 months of age)

In this task (Golub et al. 2005a, b, 2007), a screen was moved aside in $2.5-\mathrm{cm}$ increments at 2-s intervals to reveal a food treat. The monkey had to wait until the reward was fully revealed (seven intervals) to retrieve it. After adaptation and training, four 10-trial blocks were conducted in a single session in the WGTA.

\section{Reward discounting test (18 months of age)}

This test, based on previous literature (Anderson et al. 2002; Anderson and Woolverton 2003; Woolverton and Anderson 2006), used an automated apparatus (Online Resource 3). The monkey could choose either the "smallfast" button which dispensed a small food reward (one raisin) without any delay, or the "large-slow" button which dispensed a large food reward (five raisins) with a delay determined by the settings for paddle rotations. After training, the delay on the "large-slow" channel was set for $5 \mathrm{~s}$ until stable performance was reached and then increased gradually until the monkey chose the "smallfast" reward on at least half of the trials during three of four successive 14-trial sessions.

\section{Continuous performance test (CPT) (14-18 months of age)}

During CPT testing, (Golub and Donald 1995; Golub et al. 1999, 2005a, b), correct (white) and incorrect (green, red) colored rectangles were presented sequentially in randomized order on a computer touch screen placed in front of the monkey's home cage. Ten-min daily sessions containing 28 correct and 56 incorrect stimuli were continued to a criterion of $75 \%$ responses to the correct stimulus (hits) during 5 sessions. Hits were rewarded with a 45-mg sugar pellet. Performance was assessed during the 5 criterion sessions as hits (touch of correct stimulus), omission errors (miss, failure to touch correct stimulus), correct omission (failure to touch incorrect stimulus) and commission error (false alarm, touch of incorrect stimulus). Percent correct responses ((hits + correct rejections $) /($ misses + false alarms + hits + correct rejections)) was also calculated.

Pharmacological responsiveness of the dopamine system (14-18 months of age)

The influence of a dopamine agonist (apomorphine, $0.2 \mathrm{mg} /$ $\mathrm{kg}$ i.m.) and a dopamine antagonist (haloperidol, $0.02 \mathrm{mg} / \mathrm{kg}$ i.m.) on responding maintained by a fixed interval (FI) schedule of reinforcement was determined (Golub et al. $2005 \mathrm{a}, \mathrm{b})$. This task was conducted using a touch screen placed in front of the home cage and was rewarded with a $45 \mathrm{mg}$ sugar pellet. Briefly, stable performance for a 2-min 
FI schedule was established over 30 training sessions followed by a series of three drug sessions over 7 days, each preceded by a vehicle (saline) session and followed by a minimum 72-h washout period: apomorphine, haloperidol, and lastly haloperidol and apomorphine combined.

\section{Statistical analysis}

Effects evaluated were prenatal diet group (ID, IS), MAOA genotype category (hi-MAOA, low-MAOA expression) and MAOA*ID interaction. Most variables were analyzed by ANOVA or RMANOVA using general linear models (JMP9.0, SAS, Carey, NC). Planned comparisons compared ID and IS groups within each MAOA genotype group. Correlations were evaluated with Pearson's correlation coefficients. The threshold for statistical significance was $p<.05$. Effects identified as a trend $(.05<p<.065)$ were also examined in some cases using the planned comparisons.

\section{Results}

Problem solving

\section{Puzzle boxes and mazes}

Monkeys solved a median of 5 boxes. There were no effects on the number of boxes solved. One monkey in the lowMAOA ID group failed to solve any of the puzzle boxes. There was an MAOA*ID interaction on sessions to criterion on box two $(F(1,15)=6.12, p=.028)$, and the most difficult box solved by all the other monkeys. Within the lowMAOA group, ID resulted in slower learning (more sessions to criterion) (low-MAOA: ID $>$ IS, $p=.011$ ).

Monkeys solved a median of 7 mazes. For mazes, the MAOA main effect was significant with the low-MAOA group solving fewer mazes $(F(1,15)=8.58, p=.010)$, and reaching criterion more slowly than the hi-MAOA group on maze three $(p=.014)$, the most difficult maze solved by all subjects. ID did not significantly influence maze performance in either genotype group, and there was no trend for poorer performance in the low-MAOA ID in comparison with the low-MAOA IS group. Thus, lowMAOA led to poorer performance of both tasks, but this was seen only in the low-MAOA ID group for puzzle boxes.

Reward sensitivity tests

\section{Reward discounting}

A significant MAOA effect was seen for number of sessions to reach the training criterion $(F(1,15)=4.70, p=.048)$, with slower learning in the low-MAOA group $(10 \pm 2$ sessions) than the high-MAOA group ( $4 \pm 2$ sessions). The MAOA*ID interaction was not significant, but learning was slowest in the low-MAOA ID group (14 \pm 4 sessions). Reward discounting criterion was half or fewer trials where the small-fast reward was selected in three of 4 successive sessions. Most of the monkeys switched from selecting the large-slow to the small-fast reward but some (5/20) stopped responding to either button. This happened chiefly (4/5 monkeys) when they persisted in selecting the large-slow reward with delays over $100 \mathrm{~s}$. These monkeys were given a maximum score of 42 sessions. The low-MAOA group showed reward discounting in a lower mean number of sessions than the hi-MAOA group, but the difference was not significant ( $p=.07$ ). Low-MAOA ID monkeys showed higher numbers of balks (no response) as delays increased above $24 \mathrm{~s}$. The index of reward discounting, the number of slow responses as the delay interval increased, was also lowest in the low-MAOA ID group but not significantly different from the hi-MAOA ID group $(p=.11)$.

\section{Reward preference and devaluation}

The low-MAOA ID group had a lower number of sessions to criterion in learning the concurrent discrimination than the other groups ( $27 \pm 7$ vs. $44 \pm 4, p=.039$ ). During the preference sessions, the reward value of the preferred food item prior to satiation showed an MAOA*ID interaction $(F(3,11)=8.45, \quad p=.0143, \quad$ ID: $\quad$ low-MAOA $<$ hiMAOA, $p=.007$ ) (Fig. 1a). The opposite trend was seen for response latencies; during probe sessions, the hiMAOA ID group had consistently shorter latencies than the other groups $(p=.025)$.

Satiation with either the preferred $(p<.0001)$ or nonpreferred $(p=.0006)$ reward decreased preference for the preferred reward (reward devaluation) with no change in preference for non-preferred reward (data not shown). In our young monkeys, satiation also increased the number of trials during which no choice was made. There were no effects of MAOA or ID on reward devaluation.

In summary, all the monkeys showed a strong preference for one of the two rewards and also showed reward devaluation. The hi-MAOA ID monkeys showed greater preference and faster response to reward than low-MAOA monkeys suggesting stronger reward dependence. There were no differences in reward devaluation; however, differential devaluation after satiation was not seen in the sample as a whole.

\section{Reward delay}

Highly variable performances with no consistent MAOA or ID effects were seen. Although there were no significant 
Fig. 1 Performance of cognitive tests reflecting interactions of MAOA genotype (low or high transcription rates) and dietary iron (iron sufficient (IS) or iron deficient (ID). a, b Performance is similar in the two genotype groups with an iron-sufficient diet, but diverges with iron deficiency.

c, d Performance differs in the two genotype groups with an iron-sufficient diet but converges with iron deficiency. In all 4 tasks, the direction of the effect of ID is consistent within genotype group, increasing performance measures in the hi-MAOA group and decreasing them in the low-MAOA group. * indicates statistically significant genotype by ID interaction

\section{a Reward devaluation: strength of preference}

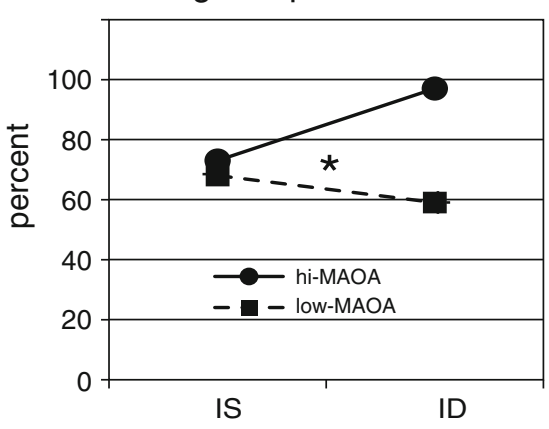

c CPT correct choices

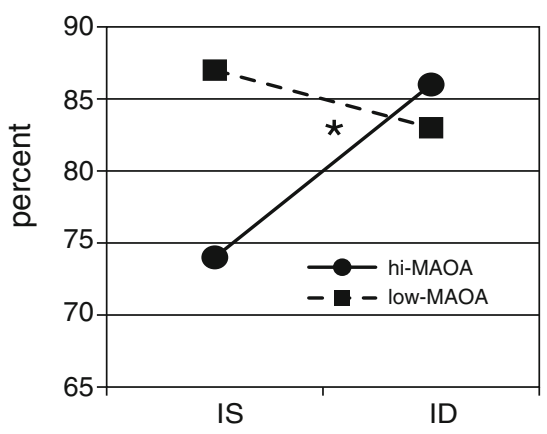

b Fixed Interval rewards

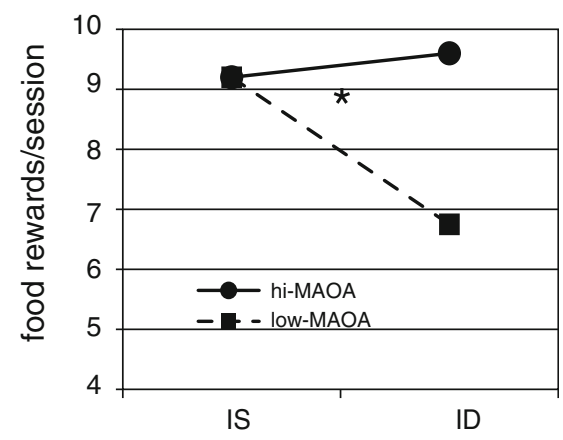

d Reward delay

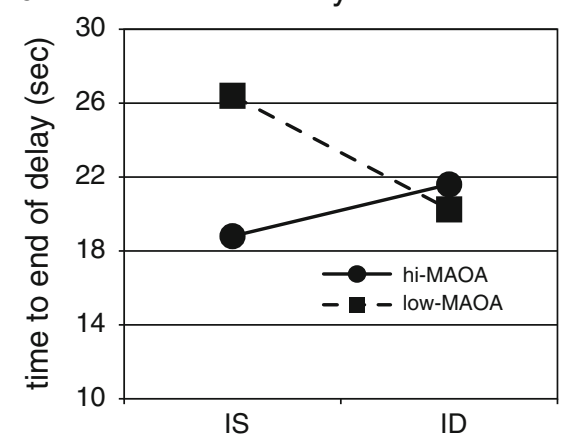

effects for response latency across the session, the pattern of means suggested greater impulsivity in the hi-MAOA group under control diet (IS) conditions (Fig. 1d).

\section{Continuous performance test}

There were no effects on the number of sessions needed to reach criterion for responding reliably to the positive stimulus. An MAOA*ID interaction was seen for percent correct responses in the final five criterion sessions of the monkeys that reached criterion $(F(1,12)=5.13$, $p=.0428$, hi-MAOA: ID > IS, $p=.029$ ) (Fig. 1c). The number of false alarms during these sessions also showed an MAOA*ID interaction $(F(1,12)=5.05, p=.044)$ with a lower number of false alarms in the hi-MAOA ID versus IS group $(p=.026)$ (data not shown). Although the MAOA main effect was not significant $(p=.12)$, within the IS group, the hi-MAOA monkeys had higher commission errors than the low-MAOA group $(p=.02)$. This indication of greater impulsivity in the hi-MAOA genotype monkeys was similar to the findings for Reward Delay (Fig. 1c, d).

\section{Pharmacological response of dopamine system}

The low-MAOA ID group had poorer performance throughout the series of vehicle and drug sessions. An MAOA*ID interaction was seen for the average number of reinforcements obtained during the vehicle sessions $(F(1,15)=7.28, \quad p=.017, \quad$ low-MAOA $\quad$ ID $<$ IS, $p=.009$ ) (Fig. 1b). The number of responses during vehicle sessions showed a similar pattern (Table 2). In fact, one low-MAOA ID monkey had to be removed from the drug challenge analysis due to complete lack of responding during some vehicle sessions. There were no statistically significant effects on performance during the apomorphine sessions. The depression in responding during haloperidol sessions was minimal in the low-MAOA ID group; however, the lower baseline response level needs to be considered (Table 2). Apomorphine alleviation of haloperidol depressed responding showed a marginally significant main effect of ID in the ANOVA $(F(1,18)=4.61, p=.046)$. Again, the low response baseline of the low-MAOA ID subjects complicates interpretation.

\section{Discussion}

Cognitive deficits, primarily identified by standardized scales of infant cognitive development and childhood IQ tests, are known to be associated with developmental iron deficiency in infants and children (Lozoff et al. 2008; Peirano et al. 2009). More recently, iron supplementation during pregnancy and the early postpartum period has been found to predict improved performance in standardized IQ 
Table 2 Pharmacological response of the dopamine system

\begin{tabular}{|c|c|c|c|c|}
\hline & \multicolumn{2}{|l|}{ Hi-MAOA } & \multicolumn{2}{|l|}{ Low-MAOA } \\
\hline & IS & ID & IS & ID \\
\hline Vehicle session: reinforcements* & $9.1 \pm 0.1^{\mathrm{a}}$ & $9.3 \pm 0.2$ & $9.3 \pm 0.2$ & $8.0 \pm 0.7 * *$ \\
\hline Vehicle session: responses & $184 \pm 37$ & $175 \pm 20$ & $181 \pm 38$ & $87 \pm 8$ \\
\hline Haloperidol session: change in number of responses & $-163 \pm 48$ & $-151 \pm 31$ & $-141 \pm 48$ & $-1 \pm 29$ \\
\hline Haloperidol + apomorphine: change in number of responses*** & $-149 \pm 51$ & $-79 \pm 21$ & $-124 \pm 32$ & $-67 \pm 26$ \\
\hline
\end{tabular}

See text for interpretation

* Significant MAOA by ID interaction by ANOVA, $p<0.02$

** Significant IS/ID post hoc comparison within low-MAOA group, $p<0.01$

*** Significant ID effect by ANOVA, $p<.05$

${ }^{\text {a }}$ Mean \pm sem

Table 3 Description of findings from the cognitive test battery in juvenile monkeys

\begin{tabular}{|c|c|c|c|}
\hline & MAOA main effect & Diet effects in low-MAOA group & Diet effects in hi-MAOA group \\
\hline Reward devaluation (8-11 months) & $\begin{array}{l}\text { Slower learning in } \\
\text { low-MAOA }\end{array}$ & $\begin{array}{l}\text { Lower reward value of preferred food in } \\
\text { ID than IS }\end{array}$ & \\
\hline \multicolumn{4}{|l|}{ Reward delay } \\
\hline \multicolumn{4}{|l|}{ Reward discounting } \\
\hline $\begin{array}{l}\text { Fixed interval performance } \\
\text { (14-18 months) }\end{array}$ & & $\begin{array}{l}\text { Fewer reinforcements obtained in ID } \\
\text { than IS }\end{array}$ & \\
\hline $\begin{array}{l}\text { Continuous performance test } \\
\text { (14-18 months) }\end{array}$ & & & $\begin{array}{l}\text { Fewer false alarms in ID than } \\
\text { IS group }\end{array}$ \\
\hline Puzzles (16-18 months) & & Slower learning in ID than IS & \\
\hline Mazes (16-18 months) & $\begin{array}{l}\text { Fewer mazes solved in } \\
\text { low-MAOA }\end{array}$ & & \\
\hline
\end{tabular}

and executive function testing of school age children in Nepal (Christian et al. 2010). While this research suggests a link between prenatal ID and cognitive performance, the present non-human primate studies offer a more direct, controlled investigation of this causal relationship. Findings are summarized in Table 3 . The study supports a strong interaction between MAOA polymorphism and prenatal ID on task performance, and an underlying disruption of reward dependence.

The tests reported here involved food-rewarded learning and performance. As was the case in a previous experiment, ID alone did not influence these tests. However, in parallel with previous evaluation of response to social challenge, ID did influence learning and performance in monkeys with low-MAOA polymorphism genotypes. In several rewarded tasks, poorer performance levels were seen in the low-MAOA ID group than the other three groups. Examples are more sessions to criterion for boxes and mazes, fewer rewards obtained under the Fixed
Interval schedule, and more sessions to criterion for learning Reward Discounting (see Table 3).

An insight into different performance levels in rewarded tasks may be gleaned from the probe sessions of the Reward Devaluation test. ID led to a stronger reward preference and shorter response latencies in the hi-MAOA but weaker preference in the low-MAOA monkeys. Weaker reward strength may have led to generally lowered performance in the low-MAOA ID group. Low-MAOA genotypes in men have been shown to be associated with less reward dependence, a trait determined from questionnaires, as well as increased functional connectivity between ventromedial prefrontal cortex and the amygdala (Buckholtz et al. 2008). Reward efficacy testing is not available in human studies of prenatal or postnatal ID. However, many effects of developmental ID have been attributed to dopamine mediation, and dopamine is known to be involved in cerebral reward systems (Lozoff 2011). Unfortunately few conclusions could be drawn from the 
test of Pharmacological Response of the Dopamine System in the current study because of group differences in baseline performance. Further examination of reward systems with behavioral and imaging approaches may help define a reward basis for MAOA*ID interactions influencing cognitive performance.

Although prenatal ID effects may differ in direction depending on genotype in this study (Fig. 1), it is still possible to posit an additive effect of MAOA and ID mediated by the dopamine system. Bell-shaped, or inverted-U-shaped, activation-effect curves are associated with prefrontal dopamine receptor activation and cognition, recently attributed to neuronal network dynamics (Stewart and Plenz 2006) or intracellular signaling pathways (Vijayraghavan et al. 2007).

The Continuous Performance Test (CPT) suggested an interesting MAOA genotype finding. The control (IS) hiMAOA group made more errors of commission ("false alarms") than the control low-MAOA group. There is a growing literature concerning MAOA polymorphisms and attention deficit hyperactivity disorder (ADHD), generally using diagnostic categories or clinical evaluation tools, rather than performance tests like the CPT (Liu et al. 2011). One study, however, found higher commission errors in boys with ADHD and hi-MAOA polymorphisms compared to low-MAOA polymorphisms (Manor et al. 2002). In our study, although the MAOA main effect was not significant ( $p=.12$ ), within the control diet group (IS), the hi-MAOA monkeys had higher commission errors than the lowMAOA group $(p=.02)$. Another test reflecting impulsivity, the Reward Delay test, demonstrated lower mean latencies (more impulsive) in the hi-MAOA IS group, although group differences were not significant. Better task performance in terms of fewer false alarms and longer reward delay latencies could be interpreted as better response inhibition but also less reward sensitivity in the low-MAOA ID group (Solanto et al. 2001; Vaidya et al. 2004; Winstanley et al. 2004).

Because of the plethora of iron-dependent enzymes, there are many downstream locations where different MAOA transcription rates could interact with ID during brain development. Prominent among these are the monoamine neurotransmitter synthesis pathways, which contain iron-dependent enzymes tyrosine and tryptophan hydroxylase, the adrenal, where iron-dependent cytochrome enzymes synthesize cortisol, and the mitochondria, with high levels of iron as well as MAOA oxidative activity (Lu and Cortopassi 2007; Kakhlon et al. 2008).

This experiment involved a small group of monkeys and a large number of tests under tightly controlled environmental conditions. Both specific findings and general conclusions require replication and extension, including use of both sexes, larger sample sizes, testing at later ages and investigations to support biological links between genotype and prenatal ID. More focused testing in more sensitive experiments with larger sample sizes may also uncover additional functional endpoints that aid in interpretation.

To what extent are the MAOA*ID interactions described here of relevance to humans? The prevalence of MAOA VNTR polymorphism varies with the human population studied, but commonly favors the high transcription alleles $(60: 40)$ to the extent that the range of polymorphisms and their transcription rates are known (Sabol et al. 1998). Because MAOA is X-linked, a phenotype attributable to high versus low transcription will occur at a rate proportional to the allele distribution in males, but at a much lower rate in females, since only females homozygous for a high or low transcription VNTR polymorphism will be expected to show the phenotype. Recent data indicate that $34 \%$ of pregnant women in the US experience iron deficiency anemia during the third trimester (Bashiri et al. 2003; Centers for Disease Control 2011). Thus, combined nutrient and genotype risk may be a significant determinant of cognitive performance in children.

Acknowledgments The authors appreciate the expert work of Alicia Bulleri, who conducted behavioral testing, and the animal technical staff at CNPRC. This work was supported by National Institutes of Health Grants OD011107 (Harris Lewin, PI) to the California National Primate Research Center, and P01HD39386 (Betsy Lozoff, PI; Mari Golub Project 2 PI).

\section{References}

Anderson KG, Woolverton WL (2003) Effects of dose and infusion delay on cocaine self-administration choice in rhesus monkeys. Psychopharmacology 167:424-430

Anderson KG, Velkey AJ, Woolverton WL (2002) The generalized matching law as a predictor of choice between cocaine and food in rhesus monkeys. Psychopharmacology 163:319-326

Bashiri A, Burstein E, Sheiner E, Mazor M (2003) Anemia during pregnancy and treatment with intravenous iron: review of the literature. Eur J Obstet Gynecol Reprod Biol 110:2-7

Buckholtz JW, Callicott JH, Kolachana B, Hariri AR, Goldberg TE, Genderson M, Egan MF, Mattay VS, Weinberger DR, MeyerLindenberg A (2008) Genetic variation in MAOA modulates ventromedial prefrontal circuitry mediating individual differences in human personality. Mol Psychiatry 13:313-324

Capitanio JP, Del Rosso LA, Calonder LA, Blozis SA, Penedo MC (2012) Behavioral effects of prenatal ketamine exposure in rhesus macaques are dependent on MAOA genotype. Exp Clin Psychopharmacol 20:173-180

Centers for Disease Control (2011) CDC's pediatric and pregnancy nutrition surveillance system 2010. http://www.cdc.gov/pednss/ pnss_tables/html/pnss_national_table5.htm

Chang S, Zeng L, Brouwer ID, Kok FJ, Yan H (2013) Effect of iron deficiency anemia in pregnancy on child mental development in rural China. Pediatrics 131:e755-e763 
Christian P, Murray-Kolb LE, Khatry SK, Katz J, Schaefer BA, Cole PM, Leclerq SC, Tielsch JM (2010) Prenatal micronutrient supplementation and intellectual and motor function in early school-aged children in Nepal. JAMA 304:2716-2723

Cicchetti D, Rogosch FA, Thibodeau EL (2012) The effects of child maltreatment on early signs of antisocial behavior: genetic moderation by tryptophan hydroxylase, serotonin transporter, and monoamine oxidase A genes. Dev Psychopathol 24:907-928

Derringer J, Krueger RF, Irons DE, Iacono WG (2010) Harsh discipline, childhood sexual assault, and MAOA genotype: an investigation of main and interactive effects on diverse clinical externalizing outcomes. Behav Genet 40:639-648

Fergusson DM, Boden JM, Horwood LJ, Miller A, Kennedy MA (2012) Moderating role of the MAOA genotype in antisocial behaviour. Br J Psychiatry 200:116-123

Golub MS, Donald JM (1995) Effect of intrapartum meperidine on behavior of 3- to 12-month-old infant rhesus monkeys. Biol Neonate 67:140-148

Golub MS, Keen CL, Gershwin ME (1999) Behavioral and hematologic consequences of marginal iron-zinc nutrition in adolescent monkeys and the effect of a powdered beef supplement. Am J Clin Nutr 70:1059-1068

Golub MS, Hogrefe CE, Germann SL, Capitanio JL, Lozoff B (2005a) Behavioral consequences of developmental iron deficiency in infant rhesus monkeys. Neurotoxicol Teratol 28:3-17

Golub MS, Hogrefe CE, Germann SL, Tran TT, Beard JL, Crinella FM, Lonnerdal B (2005b) Neurobehavioral evaluation of rhesus monkey infants fed cow's milk formula, soy formula, or soy formula with added manganese. Neurotoxicol Teratol 27:615-627

Golub MS, Hogrefe CE, Tarantal AF, Germann SL, Beard JL, Calatroni A (2006) Diet-induced iron deficiency anemia and pregnancy outcome in the rhesus monkey. Am J Clin Nutr 83:647-656

Golub MS, Hogrefe CE, Germann SL (2007) Iron deprivation during fetal development changes the behavior of juvenile rhesus monkeys. J Nutr 137:979-984

Golub MS, Hogrefe CE, Widaman KF, Capitanio JP (2009) Iron deficiency anemia and affective response in rhesus monkey infants. Dev Psychobiol 51:47-59

Golub MS, Hogrefe CE, Unger EL (2012) Influence of prenatal iron deficiency and MAOA genotype on response to social challenge in rhesus monkey infants. Genes Brain Behav 11:278-290

Hill J, Breen G, Quinn J, Tibu F, Sharp H, Pickles A (2013) Evidence for interplay between genes and maternal stress in utero: monoamine oxidase A polymorphism moderates effects of life events during pregnancy on infant negative emotionality at 5 weeks. Genes Brain Behav 12:388-396

Jimenez DF, Tarantal AF (2003) Fetal gender determination in early first trimester pregnancies of rhesus monkeys (Macaca mulatta) by fluorescent PCR analysis of maternal serum. J Med Primatol 32:315-319

Kakhlon O, Manning H, Breuer W, Melamed-Book N, Lu C, Cortopassi G, Munnich A, Cabantchik ZI (2008) Cell functions impaired by frataxin deficiency are restored by drug-mediated iron relocation. Blood 112:5219-5227

Kim-Cohen J, Caspi A, Taylor A, Williams B, Newcombe R, Craig IW, Moffitt TE (2006) MAOA, maltreatment, and geneenvironment interaction predicting children's mental health: new evidence and a meta-analysis. Mol Psychiatry 11:903-913

Kinnally EL, Huang YY, Haverly R, Burke AK, Galfalvy H, Brent DP, Oquendo MA, Mann JJ (2009) Parental care moderates the influence of MAOA-uVNTR genotype and childhood stressors on trait impulsivity and aggression in adult women. Psychiatr Genet 19:126-133

Liu L, Guan LL, Chen Y, Ji N, Li HM, Li ZH, Qian QJ, Yang L, Glatt SJ, Faraone SV, Wang YF (2011) Association analyses of
MAOA in Chinese Han subjects with attention-deficit/hyperactivity disorder: family-based association test, case-control study, and quantitative traits of impulsivity. Am J Med Genet B Neuropsychiatr Genet 156B:737-748

Lozoff B (2011) Early iron deficiency has brain and behavior effects consistent with dopaminergic dysfunction. J Nutr 141:740S-746S

Lozoff B, Clark KM, Jing Y, Armony-Sivan R, Angelilli ML, Jacobson SW (2008) Dose-response relationships between iron deficiency with or without anemia and infant social-emotional behavior. J Pediatr 152:696-702

Lu C, Cortopassi G (2007) Frataxin knockdown causes loss of cytoplasmic iron-sulfur cluster functions, redox alterations and induction of heme transcripts. Arch Biochem Biophys 457:111-122

Machado CJ, Bachevalier J (2007) The effects of selective amygdala, orbital frontal cortex or hippocampal formation lesions on reward assessment in nonhuman primates. Eur $\mathbf{J}$ Neurosci 25:2885-2904

Malkova L, Gaffan D, Murray EA (1997) Excitotoxic lesions of the amygdala fail to produce impairment in visual learning for auditory secondary reinforcement but interfere with reinforcer devaluation effects in rhesus monkeys. J Neurosci 17:6011-6020

Manor I, Tyano S, Mel E, Eisenberg J, Bachner-Melman R, Kotler M, Ebstein RP (2002) Family-based and association studies of monoamine oxidase A and attention deficit hyperactivity disorder (ADHD): preferential transmission of the long promoterregion repeat and its association with impaired performance on a continuous performance test (TOVA). Mol Psychiatry 7:626-632

Newman TK, Syagailo YV, Barr CS, Wendland JR, Champoux M, Graessle M, Suomi SJ, Higley JD, Lesch KP (2005) Monoamine oxidase A gene promoter variation and rearing experience influences aggressive behavior in rhesus monkeys. Biol Psychiatry $57: 167-172$

Nymberg C, Jia T, Lubbe S, Ruggeri B, Desrivieres S, Barker G, Buchel C, Fauth-Buehler M, Cattrell A, Conrod P, Flor H, Gallinat J, Garavan H, Heinz A, Ittermann B, Lawrence C, Mann K, Nees F, Salatino-Oliveira A, Paillere Martinot M L, Paus T, Rietschel M, Robbins T, Smolka M, Banaschewski T, Rubia K, Loth E, Schumann G (2013) Neural mechanisms of attentiondeficit/hyperactivity disorder symptoms are stratified by MAOA genotype. Biol Psychiatry 74:607-614

Peirano PD, Algarin CR, Chamorro R, Reyes S, Garrido MI, Duran S, Lozoff B (2009) Sleep and neurofunctions throughout child development: lasting effects of early iron deficiency. J Pediatr Gastroenterol Nutr 48(Suppl 1):S8-15

Sabol SZ, Hu S, Hamer D (1998) A functional polymorphism in the monoamine oxidase A gene promoter. Hum Genet 103:273-279

Solanto MV, Abikoff H, Sonuga-Barke E, Schachar R, Logan GD, Wigal T, Hechtman L, Hinshaw S, Turkel E (2001) The ecological validity of delay aversion and response inhibition as measures of impulsivity in AD/HD: a supplement to the NIMH multimodal treatment study of AD/HD. J Abnorm Child Psychol 29:215-228

Stewart CV, Plenz D (2006) Inverted-U profile of dopamine-NMDAmediated spontaneous avalanche recurrence in superficial layers of rat prefrontal cortex. J Neurosci 26:8148-8159

Tsuchida J, Kawasaki K, Sankai T, Kubo N, Terao K, Koyama T, Makino J, Yoshikawa Y (2003) New type of puzzle-task finger maze learning in Macaca fascicularis. Int J Primatol 24:261-270

Vaidya JG, Grippo AJ, Johnson AK, Watson D (2004) A comparative developmental study of impulsivity in rats and humans: the role of reward sensitivity. Ann N Y Acad Sci 1021:395-398

Vijayraghavan S, Wang M, Birnbaum SG, Williams GV, Arnsten AF (2007) Inverted-U dopamine D1 receptor actions on prefrontal neurons engaged in working memory. Nat Neurosci 10:376-384 
West EA, DesJardin JT, Gale K, Malkova L (2011) Transient inactivation of orbitofrontal cortex blocks reinforcer devaluation in macaques. J Neurosci 31:15128-15135

Winstanley CA, Dalley JW, Theobald DE, Robbins TW (2004) Fractionating impulsivity: contrasting effects of central 5-HT depletion on different measures of impulsive behavior. Neuropsychopharmacology 29:1331-1343

Woolverton WL, Anderson KG (2006) Effects of delay to reinforcement on the choice between cocaine and food in rhesus monkeys. Psychopharmacology 186:99-106 